ISSN 1980-5098

\title{
VELOCIDADE DE CARBOXILAÇÃO DA RUBISCO E TRANSPORTE DE ELÉTRONS EM ESPÉCIES ARBÓREAS EM RESPOSTA A FATORES DO AMBIENTE NA AMAZÔNIA CENTRAL
}

\author{
MAXIMUM CARBOXYLATION VELOCITY OF RUBISCO AND MAXIMUM RATE OF \\ ELECTRON TRANSPORT IN SAPLINGS IN RESPONSE TO VARIATIONS IN ENVIRONMENTAL \\ FACTORS IN CENTRAL AMAZONIA
}

Keila Rêgo Mendes ${ }^{1}$ Ricardo Antonio Marenco ${ }^{2}$ Helena Cristina Santos Nascimento ${ }^{3}$

\begin{abstract}
RESUMO
A disponibilidade de luz é um dos fatores que mais limita a fotossíntese de árvores juvenis no sub-bosque da floresta. Os objetivos deste estudo foram determinar como os parâmetros fotossintéticos (velocidade máxima de carboxilação da Rubisco - Vc-max e as taxas máximas de transporte de elétrons - Jmax) respondem à irradiância, temperatura e horário do dia no interior da floresta. Os dados foram coletados nos meses de setembro a novembro de 2010 , entre $08 \mathrm{~h} 00$ e $16 \mathrm{~h} 00$, em condições ambientais de temperatura $\left(27 \pm 2^{\circ} \mathrm{C}\right)$ e umidade relativa do ar $(70 \pm 3 \%)$. Fotossíntese saturada por $\mathrm{CO}_{2}\left(\mathrm{~A}_{\text {pot }}\right)$ foi mensurada em luz saturante e $\left[\mathrm{CO}_{2}\right]$ de $2000 \mu \mathrm{mol} \mathrm{mol}^{-1}$. Os dados foram submetidos à análise de variância, o teste de Tukey foi utilizado para separação de médias. $\mathrm{V}_{\mathrm{c} \text {-max } \mathrm{e}} \mathrm{J}_{\max }$ correlacionaram com a ffotossíntese em [CO2] de 380 $\mu$ mol mol-1 $\left(\mathrm{A}_{380}\right)$ e $\mathrm{A}_{\text {pot }} \mathrm{O}$ aumento de $\mathrm{C}_{\mathrm{i}}$ causou o aumento da fotossíntese, até o ponto da limitação da fotossíntese por $\mathrm{J}_{\max }$. Abaixo do ponto de colimitação $\left(300\right.$ a $\left.569 \mu \mathrm{mol} \mathrm{mol}^{-1}\right)$, as taxas fotossintéticas foram limitadas por $\mathrm{V}_{\text {c-max }}$. Aumentos da irradiância e temperatura no sub-bosque aumentaram os valores de $\mathrm{A}_{380}$, $\mathrm{A}_{\mathrm{pot}}, \mathrm{V}_{\mathrm{c} \text {-max }} \mathrm{e} \mathrm{J}_{\text {max }}$. Entretanto, não houve efeito significativo $(p>0,05)$ do horário do dia em $\mathrm{V}_{\mathrm{c} \text {-max }} \mathrm{e} \mathrm{J}_{\max }$. Ausência de efeito do horário do dia é importante para a folha poder utilizar com alta eficiência os feixes de luz direta que ocasionalmente atingem o sub-bosque da floresta.
\end{abstract}

Palavras-chave: limitação não estomática; colimitação da fotossíntese; variação diurna.

\section{ABSTRACT}

Light availability is one of the main factors that limits photosynthetic rates in saplings in the forest understory. The aims of this study were to determine how photosynthetic parameters (maximum carboxylation velocity of Rubisco $-\mathrm{V}_{\mathrm{c}-\max }$ and maximum rate of electron transport $-\mathrm{J}_{\max }$ ) respond to irradiance, temperature and diurnal variations in the physical environment. Gas exchange data were collected between $08 \mathrm{~h} 00$ and $16 \mathrm{~h} 00$, and from September to November (2010) under ambient conditions, temperature of $27 \pm 2{ }^{\circ} \mathrm{C}$ and relative humidity of $70 \pm 3 \% . \mathrm{CO}_{2}$ saturated photosynthesis $\left(\mathrm{A}_{\mathrm{pot}}\right)$ was measured under light saturation and $\left[\mathrm{CO}_{2}\right]$ of $2000 \mu \mathrm{mol} \mathrm{mol}^{-1}$. Data were subjected to analysis of variance and then the Tukey test was used for mean separations. $\mathrm{V}_{\mathrm{c}-\max }$ and $\mathrm{J}_{\max }$ correlated with photosynthetic rates at $\left[\mathrm{CO}_{2}\right]$ of $380 \mu \mathrm{mol} \mathrm{mol}^{-1}\left(\mathrm{~A}_{380}\right)$ and $\mathrm{A}_{\text {pot }}$. The increase in intercellular $\mathrm{CO}_{2}$ concentration $\left(\mathrm{C}_{\mathrm{i}}\right)$ led to increase in photosynthetic rates, up to the point of photosynthesis limitation by the electron transport rate. Below the collimation point (300-569 $\mu \mathrm{mol} \mathrm{mol}^{-1)}$ photosynthetic rates were limited by $\mathrm{V}_{\mathrm{c}-\mathrm{max}}$. Increases in irradiance and temperature in the forest understory led to increasing the values of $\mathrm{A}_{380}, \mathrm{~A}_{\mathrm{pot}}, \mathrm{V}_{\mathrm{c}-\max }$ and $\mathrm{J}_{\max }$. However, the effect of time of day on

1 Bióloga, Dra ., Programa de Pós-graduação em Botânica, Instituto Nacional de Pesquisas da Amazônia, Av.André Araújo, 2936, CEP 69067-375, Manaus (AM), Brasil. keilastm@hotmail.com

2 Agrônomo, Dr., Coordenação de Dinâmica Ambiental, Instituto Nacional de Pesquisas da Amazônia, Av. André Araújo, 2936, CEP 69067-375, Manaus (AM), Brasil.rmarenco@inpa.gov.br

3 Bióloga, Dra ., Programa de Pós-graduação em Botânica, Instituto Nacional de Pesquisas da Amazônia, Av. André Araújo, 2936, CEP 69067-375, Manaus (AM), Brasil. hc_crys@hotmail.com 
$\mathrm{V}_{\mathrm{c}-\max }$ and $\mathrm{J}_{\max }$ was not significant $(p>0.05)$. Absence of an effect of time of day on $\mathrm{V}_{\mathrm{c}-\max }$ and $\mathrm{J}_{\max }$ is important for the leaf to efficiently use the sun flecks that occasionally puncture the forest floor.

Keywords: nonstomatal limitations; collimation of photosynthesis; diurnal variation.

\section{INTRODUÇÃO}

Nas florestas tropicais úmidas, a radiação fotossinteticamente ativa ao longo do perfil da floresta é muito variada, com pouca luz atingindo o sub-bosque, o que influencia a capacidade fotossintética de árvores na fase juvenil no seu ambiente natural (FETCHER; OBERBAUER; CHAZDON, 1994). Além disso, a disponibilidade de luz no sub-bosque da floresta também pode ser um fator limitante para o crescimento das árvores na fase juvenil (LOPES-TOLEDO et al., 2008). Em comparação com plantas de sol, plantas de sombra apresentam respostas morfológicas e fisiológicas diferentes (BOARDMAN, 1977); baixa irradiância muitas vezes leva a uma diminuição na espessura da folha e na massa foliar específica (VALLADARES; NIINEMETS, 2008).

A capacidade fotossintética de uma folha depende de fatores difusivos (i.e. condutância estomática e condutância do mesofilo) e não difusivos (i.e. cinética da enzima Rubisco (ribulose-1,5-bisfosfato carboxilase/oxigenase [EC 4.1.1.39] e taxa de transporte de elétrons). Tem-se relatado que baixa irradiância no sub-bosque durante o crescimento das plantas limita a absorção de carbono (MENDES; MARENCO, 2010; 2014). Isso pode ocorrer devido à redução na velocidade máxima de carboxilação da Rubisco $\left(\mathrm{V}_{\mathrm{c} \text {-max }}\right)$ e na taxa máxima de regeneração da ribulose bisfosfato, $\operatorname{RuBP}\left(\mathrm{J}_{\max }\right)$, dependente do transporte de elétrons, bem como pela diminuição na taxa de difusão de $\mathrm{CO}_{2}$ para os cloroplastos (NASCIMENTO; MARENCO, 2013). Além disso, a temperatura tem efeito direto e indireto na fotossíntese (WAY; OREN, 2010). O efeito indireto refere-se ao fechamento dos estômatos o que contribui para reduzir a perda de água via transpiração. Por outro lado, o efeito direto da temperatura na fotossíntese está associado às mudanças na atividade da Rubisco, bem como à regeneração da RuBP no ciclo de Calvin (LLOYD; FARQUHAR, 2008).

Tendo em vista que vários modelos climáticos (p.ex. SiB2, CLM; SELLERS et al., 1997) utilizam dados da velocidade máxima de carboxilação da Rubisco $\left(\mathrm{V}_{\text {c-max }}\right)$ e das taxas máximas de transporte de elétrons ( $J_{\max }$ uma função da radiação fotossinteticamente ativa) torna-se importante o estudo destes parâmetros em espécies amazônicas. Alguns estudos relatam os efeitos das variações na abertura do dossel nas taxas fotossintéticas e condutância estomática em plantas jovens da Amazônia (NASCIMENTO; MARENCO, 2013; MARENCO; NASCIMENTO; MAGALHÃES, 2014; MARENCO et al., 2015). Na Amazônia central, Mendes e Marenco (2010) observaram que o funcionamento dos estômatos e fotossíntese de algumas espécies responde à variação diurna dos fatores ambientais. Entretanto, há falta de informação sobre os efeitos de fatores do ambiente físico (irradiância e temperatura) e do horário do dia em $\mathrm{J}_{\max } \mathrm{e} \mathrm{V}_{\mathrm{c} \text {-max }}$ em árvores da Amazônia, particularmente para árvores na fase juvenil que crescem no sub-bosque. Neste estudo partiu-se da premissa de que a variação da irradiância e temperatura do ar no sub-bosque afetam $\mathrm{V}_{\mathrm{c} \text { - }}$ ${ }_{\max } \mathrm{e}_{\max }$ de árvores na fase juvenil em seu ambiente natural. Além disso, foi testada a hipótese que $\mathrm{V}_{\mathrm{c}-\max } \mathrm{e}$ $\mathrm{J}_{\max }$ das espécies estudadas seguem um padrão diurno. Assim, os objetivos deste estudo foram determinar como parâmetros fotossintéticos $\left(\mathrm{V}_{\mathrm{c} \text {-max }}\right.$ e $\left.\mathrm{J}_{\max }\right)$ respondem à irradiância e temperatura do ambiente físico, bem como verificar o efeito do horário do dia nos parâmetros supracitados.

\section{MATERIAL E MÉTODOS}

\section{Local do estudo e material vegetal}

O estudo foi conduzido na Estação Experimental de Silvicultura Tropical (Núcleo ZF2), do Instituto Nacional de Pesquisas da Amazônia (INPA). A área é uma floresta nativa densa de terra firme, localizada a $60 \mathrm{~km}$ ao norte de Manaus $\left(02^{\circ} 36^{\prime} 21^{\prime}\right.$ ' S, 60 $00^{\circ} 11^{\prime}$ W). O tipo de solo predominante da região é um latossolo amarelo, com baixa fertilidade e elevada acidez ( $\mathrm{pH}$ de 4,2 a 4,5). A região apresenta características de clima equatorial úmido, com temperatura média anual de $26^{\circ} \mathrm{C}$ e precipitação anual de 2420 mm (MALHI; WRIGHT, 2004). No estudo utilizaram-se cinco espécies da Amazônia central, com quatro plantas (repetições) por espécie de 1 a $3 \mathrm{~m}$ de altura e 7-23 mm de diâmetro (a 0,5 $\mathrm{m}$ do solo). Foram 
selecionadas espécies que tivessem pelo menos quatro indivíduos dentro da área de estudo. As espécies selecionadas têm diversas utilidades conforme mostrado na Tabela 1.

TABELA 1: Famílias e importância das espécies utilizadas no estudo.

TABLE 1: Families and the importance of species used in the study.

\begin{tabular}{|c|c|c|c|}
\hline Espécies & Famílias & Importância & Referências \\
\hline $\begin{array}{l}\text { Amphirrhox longifolia (A.St.-Hil.) } \\
\text { Spreng (sinon: A. surinamensis } \\
\text { (Miq.) Eichl.) }\end{array}$ & Violaceae & $\begin{array}{l}\text { Arbusto de médio ou pequeno porte, partes } \\
\text { da planta podem ser utilizados no preparo de } \\
\text { extratos antitumorais e antibacterianos. }\end{array}$ & (ANGEL, 2004; RODRIGUES, 2013) \\
\hline Duguetia flagellaris Huber & Anonnaceae & $\begin{array}{l}\text { Árvore de médio ou pequeno porte, os frutos } \\
\text { são comestíveis, tem potencial farmacológico } \\
\text { e produz óleo essencial com atividade anti- } \\
\text { inflamatória. }\end{array}$ & $\begin{array}{l}\text { (MILLER; WANDELLI; } \\
\text { GRENAND, 1989; FECHINE et al., } \\
\text { 2002) }\end{array}$ \\
\hline Fusaea longifolia (Aubl.) Saff. & Anonnaceae & $\begin{array}{l}\text { Árvore de médio porte, produz frutos } \\
\text { comestíveis, tem potencial farmacológico e a } \\
\text { sua madeira é utilizada na construção de casas e } \\
\text { lenha. }\end{array}$ & $\begin{array}{l}\text { (CHATROU; HE, 1999; ALARCÓN, } \\
\text { 2005; TAVARES et al., 2005; } \\
\text { RESQUE, 2007) }\end{array}$ \\
\hline Guarea carinata Ducke & Meliaceae & $\begin{array}{l}\text { Árvore de médio porte, utilizada em sistemas } \\
\text { silviculturais, plantas deste gênero possuem } \\
\text { propriedades medicinais. }\end{array}$ & $\begin{array}{l}\text { (ANDRADE et al., 1976; } \\
\text { CAMACHO et al., 2001; LIMA } \\
\text { FILHO et al., 2004; JADÁN et al., } \\
\text { 2015) }\end{array}$ \\
\hline $\begin{array}{l}\text { Myrcia subsericea A. Gray } \\
\text { (sinon: Eugenia nitida } \text { Vell; } \\
\text { Gomidesia nitida (Vell.) Nied.). }\end{array}$ & Myrtaceae & $\begin{array}{l}\text { Arbusto de pequeno porte, produz frutos } \\
\text { comestíveis e óleos essenciais. }\end{array}$ & (MARTINS et al.,1999) \\
\hline
\end{tabular}

\section{Parâmetros das trocas gasosas}

As trocas gasosas foram medidas com um analisador de gás infravermelho (Li-6400, Li-Cor, NE, EUA) em quatro plantas de cada espécie e duas a três folhas por planta com bom aspecto fitossanitário, totalmente expandidas e localizadas na porção superior da copa de cada planta. Os dados foram coletados em condições ambientais de temperatura $\left(27 \pm 2^{\circ} \mathrm{C}\right)$ e umidade relativa $(70 \pm 3 \%)$. As respostas da fotossíntese à concentração intercelular de $\mathrm{CO}_{2}\left(\right.$ curvas $\mathrm{A} / \mathrm{C}_{\mathrm{i}}$ ) foram determinadas em irradiância saturante de $1000 \mu \mathrm{mol}$ $\mathrm{m}^{-2} \mathrm{~s}^{-1}$, conforme descrito previamente (MENDES; MARENCO, 2015). O valor da fotossíntese mensurada em condição saturante de $\mathrm{CO}_{2}\left(2000 \mu \mathrm{mol} \mathrm{mol}^{-1}\right)$ denominou-se $\mathrm{A}_{\text {pot }}$. A partir dos dados da curva $\mathrm{A} / \mathrm{C}_{\mathrm{i}}$ obteve-se o ponto de compensação de $\mathrm{CO}_{2}(\Gamma)$ e eficiência de carboxilação, $k_{\mathrm{x}}$ (MARENCO; GONÇALVES; VIEIRA, 2001). Além disso, obteve-se a fotossíntese em $380 \mu \mathrm{mol} \mathrm{mol}^{-1} \mathrm{de} \mathrm{CO}_{2}\left(\mathrm{~A}_{380}\right)$ para comparar com as outras variáveis mensuradas. Calculou-se a velocidade máxima de carboxilação da Rubisco $\left(\mathrm{V}_{\mathrm{c}}\right.$. ${ }_{\max }$ ) e a taxa máxima de transporte de elétrons $\left(\mathrm{J}_{\text {max }}\right.$ ) usando as equações de Farquhar (FARQUHAR; VON CAEMMERER; BERRY, 1980).

$$
\begin{aligned}
\mathrm{A}_{\mathrm{c}} & =\left[\mathrm{V}_{\mathrm{c}-\max }\left(\mathrm{C}_{\mathrm{i}}-\Gamma^{*}\right)\right] /\left[\mathrm{C}_{\mathrm{i}}+\mathrm{K}_{\mathrm{c}}\left(1+O / \mathrm{K}_{\mathrm{o}}\right)\right] \\
\mathrm{A}_{\mathrm{j}} & =\left[\mathrm{J}_{\text {max }}\left(\mathrm{C}_{\mathrm{i}}-\Gamma^{*}\right)\right] /\left(\left[4 \mathrm{C}_{\mathrm{i}}+8 \Gamma^{*}\right)\right]
\end{aligned}
$$

Em que: $A_{c}$ e $A_{j}$ são as taxas fotossintéticas líquidas limitadas pela atividade da Rubisco e taxa de transporte de elétrons $\left(\mathrm{A}_{\mathrm{j}}\right)^{\mathrm{j}}$, respectivamente. $\Gamma^{*}$ representa o ponto de compensação de $\mathrm{CO}_{2}$, na ausência da respiração e na presença de luz; $\mathrm{C}_{\mathrm{i}}$ é a concentração de $\mathrm{CO}_{2}$ nos espaços intercelulares, $O$ representa a concentração de oxigênio nos espaços intercelulares; $\mathrm{K}_{\mathrm{c}}$ e $\mathrm{K}_{\mathrm{o}}$ representam a constante de Michaelis-Menten da Rubisco para carboxilação e oxigenação, respectivamente. $\mathrm{V}_{\mathrm{c} \text {-max }} \mathrm{e} \mathrm{J}_{\max }$ são frequentemente relatados na literatura a $25^{\circ} \mathrm{C}$, por isso os dados foram padronizados para esta temperatura, conforme descrito por Medlyn et al. (1999). Para determinar o efeito do horário do dia em $\mathrm{V}_{\mathrm{c}-\max }$ e $\mathrm{J}_{\text {max }}$, os dados de fotossíntese foram coletados nos horários entre $08 \mathrm{~h} 00$ e $16 \mathrm{~h} 00$, em dias selecionados ao acaso no período de setembro a novembro de 2010. 


\section{Ambiente físico}

Dados de precipitação foram coletados utilizando um pluviômetro convencional, instalado em uma torre de observação a $40 \mathrm{~m}$ de altura, localizado próximo à área de estudo. Também foram coletados dados de irradiância, umidade do ar e temperatura do sub-bosque utilizando sensores específicos (Li-190 SA, Li-Cor, EUA; Humitter 50Y, Vaisala, Oyj, Finlândia) conectados a um datalogger (Li-1400, Li-Cor, NE, EUA) e programado para registrar os dados em intervalos de 15 minutos (RFA) e 30 minutos (umidade e temperatura).

\section{Análises estatísticas}

Os dados das variáveis estudadas foram testados quanto à normalidade (procedimento de Lilliefors) e homogeneidade da variância e, posteriormente, submetidos à análise de variância (ANOVA) para avaliar as diferenças entre espécies (delineamento inteiramente casualizado). Teste post hoc de Tukey $(p \leq 0,05)$ foi utilizado para a separação das médias. Análises de regressão foram realizadas para determinar o efeito da irradiância e temperatura nos parâmetros fotossintéticos. Para análise dos dados utilizou-se o programa estatístico SAEG 9.0 da Universidade Federal de Viçosa (UFV).

\section{RESULTADOS E DISCUSSÃO}

\section{Ambiente físico}

A precipitação média dos meses de estudo (setembro a novembro) foi de $231 \mathrm{~mm}$, o que está dentro da média relatada para a Amazônia central (MARENCO et al., 2015). Durante o estudo, a temperatura do sub-bosque oscilou entre $23^{\circ} \mathrm{C}$ à noite e $27^{\circ} \mathrm{C}$ ao meio-dia. Durante a maior parte do dia, a média da umidade relativa do ar foi superior a $80 \%$, oscilando a irradiância média do sub-bosque em torno de $10 \mu \mathrm{mol} \mathrm{m}^{-2} \mathrm{~s}^{-1}$, com variações entre um ponto e outro da floresta e com picos acima de $30 \mu \mathrm{mol} \mathrm{m}{ }^{-2} \mathrm{~s}^{-1}$ (Figura 1), devido à ocorrência de feixes de luz solar direta (sunflecks). Já a irradiância acumulada variou de 0,35 para 0,9

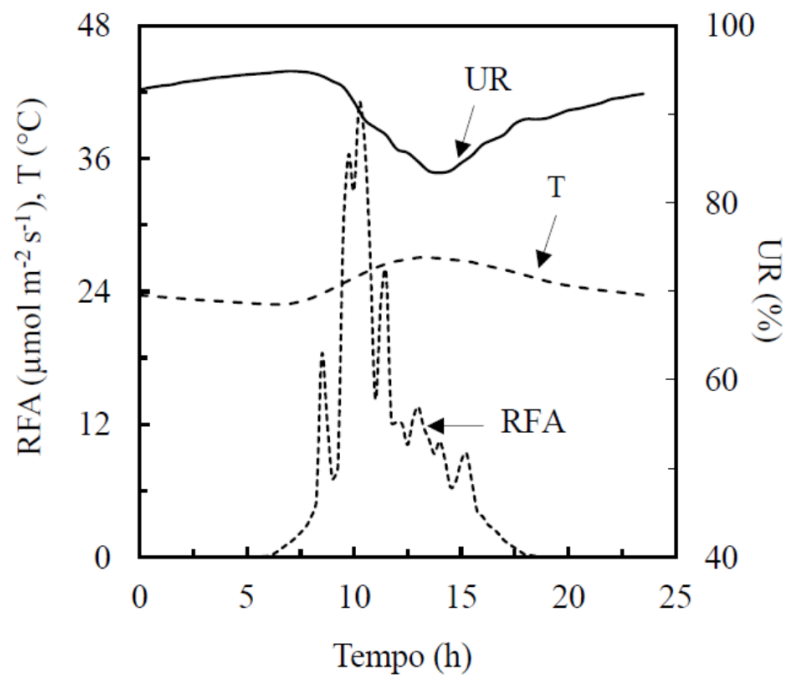

FIGURA 1: Radiação fotossinteticamente ativa (RFA, linha pontilhada), temperatura do ar ( $\mathrm{T}$, linha tracejada) e umidade relativa do ar (UR, linha sólida, eixo Y secundário, em percentagem) no sub-bosque em função do horário do dia.. Dados climáticos foram mensurados durante o período de coleta de dados de fotossíntese (setembro a novembro de 2010).

FIGURE 1: Photosynthetically active radiation (RFA, dotted line), air temperature ( $\mathrm{T}$, dashed line) and relative humidity (UR, solid line, secondary axis, in percentage) at the forest understory as a function of time of day. Climate information was collected during the period of photosynthesis data collection (September to November of 2010). 
mol m $\mathrm{m}^{-2}$ dia $^{-1}$. A irradiância média no sub-bosque da floresta foi semelhante à relatada em experimentos anteriores (MENDES; MARENCO, 2010; MENDES et al., 2013).

\section{Parâmetros das trocas gasosas}

Houve diferença significativa $(p<0,05)$ entre espécies na fotossíntese saturada por $\mathrm{CO}_{2}\left(\mathrm{~A}_{\mathrm{pot}}\right)$, condutância estomática $\left(\mathrm{g}_{\mathrm{s}[2000]}\right)$, taxa máxima de transporte de elétrons $\left(\mathrm{J}_{\max }\right)$ e velocidade máxima de carboxilação da Rubisco $\left(\mathrm{V}_{c \text {-max }}\right)$; porém, não houve efeito significativo $(p>0,05)$ entre espécies nos valores de ponto de compensação de $\mathrm{CO}_{2}(\Gamma)$ e eficiência de carboxilação $\left(k_{x}\right)$ (Tabela 2). Em média, o valor de $\mathrm{A}_{\text {pot }}$ foi $8,3 \mu \mathrm{mol} \mathrm{m} \mathrm{m}^{-2} \mathrm{~s}^{-1}$. Os valores de $\mathrm{A}_{\text {pot }}$ nas espécies estudadas são semelhantes aos encontrados por Mendes e Marenco (2010) que relatam valores de $\mathrm{A}_{\text {pot }}$ entre 7 e $9 \mu \mathrm{mol} \mathrm{m}^{-2} \mathrm{~s}^{-1}$ para espécies em condições naturais na mesma área de estudo. Os valores de $\mathrm{V}_{\mathrm{c} \text {-max }}^{\text {pot }} \mathrm{J}_{\max }$ obtidos neste estudo estão dentro da faixa de valores registrados para outras espécies da floresta tropical (WULLSCHLEGER, 1993; NASCIMENTO; MARENCO, 2013). No entanto, os valores deste estudo de $\mathrm{V}_{c \text {-max }}$ são baixos quando comparados com aqueles obtidos por Manter e Kerrigan (2004), que observaram valores entre 20 e $75 \mu \mathrm{mol} \mathrm{m} \mathrm{m}^{-2} \mathrm{~s}^{-1} \mathrm{em}$ espécies de folhas largas. Isto indica que folhas de plantas de sub-bosque adaptadas à baixa irradiância alocam pequena quantidade de assimilados e nitrogênio para síntese de proteínas da enzima Rubisco.

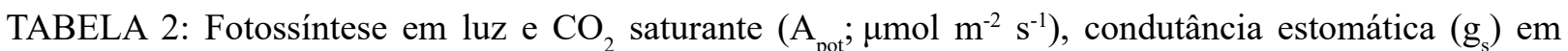
$\left[\mathrm{CO}_{2}\right]$ saturante $\left(\mathrm{g}_{\mathrm{s}[2000]} ; \mathrm{mol} \mathrm{m}^{-2} \mathrm{~s}^{-1}\right)$, velocidade máxima de carboxilação da Rubisco

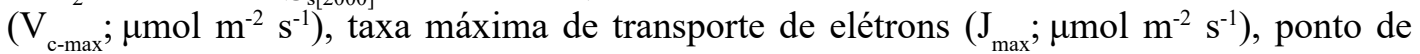
compensação de $\mathrm{CO}_{2}\left(\Gamma ; \mu \mathrm{mol} \mathrm{mol}{ }^{-1}\right)$ e eficiência de carboxilação $\left(k_{x} ; \mathrm{mmol} \mathrm{mol}^{-2} \mathrm{~s}^{-1}\right) \mathrm{em}$ cinco espécies florestais da Amazônia central. Dados coletados nos meses de setembro a novembro de 2010. Cada valor corresponde à média de quatro plantas por espécie.

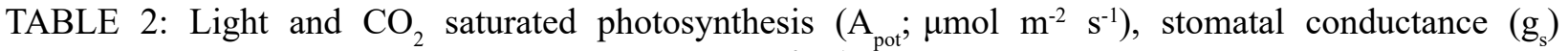
under $\left[\mathrm{CO}_{2}\right]$ saturation $\left(\mathrm{g}_{\mathrm{s}[2000]} ;\right.$ mol m $\left.\mathrm{m}^{-2} \mathrm{~s}^{-1}\right)$, maximum carboxylation velocity of Rubisco $\left(\mathrm{V}_{\mathrm{c}-\max } ; \mu \mathrm{mol} \mathrm{m} \mathrm{m}^{-2} \mathrm{~s}^{-1}\right)$, maximum electron transport rate $\left(\mathrm{J}_{\max } ; \mu \mathrm{mol} \mathrm{m} \mathrm{m}^{-2} \mathrm{~s}^{-1}\right), \mathrm{CO}_{2}$ compensation point $\left(\Gamma ; \mu \mathrm{mol} \mathrm{mol}^{-1}\right)$ and carboxylation efficiency $\left(k_{\mathrm{x}} ; \mathrm{mmol} \mathrm{m}^{-2} \mathrm{~s}^{-1}\right)$ in saplings of five species of the central Amazon. Data collected from September to November of 2010. Each value is the mean of four plants per species.

\begin{tabular}{lcccccc}
\hline \multicolumn{1}{c}{ Variáveis / Espécies } & $\mathrm{A}_{\mathrm{pot}}$ & $\mathrm{g}_{\mathrm{s}[2000]}$ & $\mathrm{V}_{\mathrm{c}-\max }$ & $\mathrm{J}_{\max }$ & $\Gamma$ & $k_{\mathrm{x}}$ \\
\hline Amphirrhox longifolia & $9,2 \mathrm{a}$ & $0,12 \mathrm{a}$ & $17,7 \mathrm{a}$ & $39,9 \mathrm{a}$ & $86,9 \mathrm{a}$ & $21,6 \mathrm{a}$ \\
Duguetia flagellaris & $8,9 \mathrm{a}$ & $0,06 \mathrm{~b}$ & $17,7 \mathrm{a}$ & $38,4 \mathrm{a}$ & $70,5 \mathrm{a}$ & $24,2 \mathrm{a}$ \\
Fusaea longifolia & $8,1 \mathrm{a}$ & $0,07 \mathrm{~b}$ & $17,6 \mathrm{a}$ & $35,4 \mathrm{ab}$ & $68,8 \mathrm{a}$ & $22,3 \mathrm{a}$ \\
Guarea carinata & $6,6 \mathrm{~b}$ & $0,09 \mathrm{ab}$ & $14,5 \mathrm{~b}$ & $25,0 \mathrm{~b}$ & $97,2 \mathrm{a}$ & $18,2 \mathrm{a}$ \\
Myrcia subsericea & $8,7 \mathrm{a}$ & $0,09 \mathrm{ab}$ & $19,4 \mathrm{a}$ & $39,0 \mathrm{a}$ & $72,9 \mathrm{a}$ & $24,5 \mathrm{a}$ \\
Média & 8,3 & 0,09 & 17,4 & 35,5 & 79,3 & 22,2 \\
\hline
\end{tabular}

Em que: Médias seguidas de mesmas letras nas colunas não diferem de acordo com o teste de Tukey a 5\% de probabilidade. 


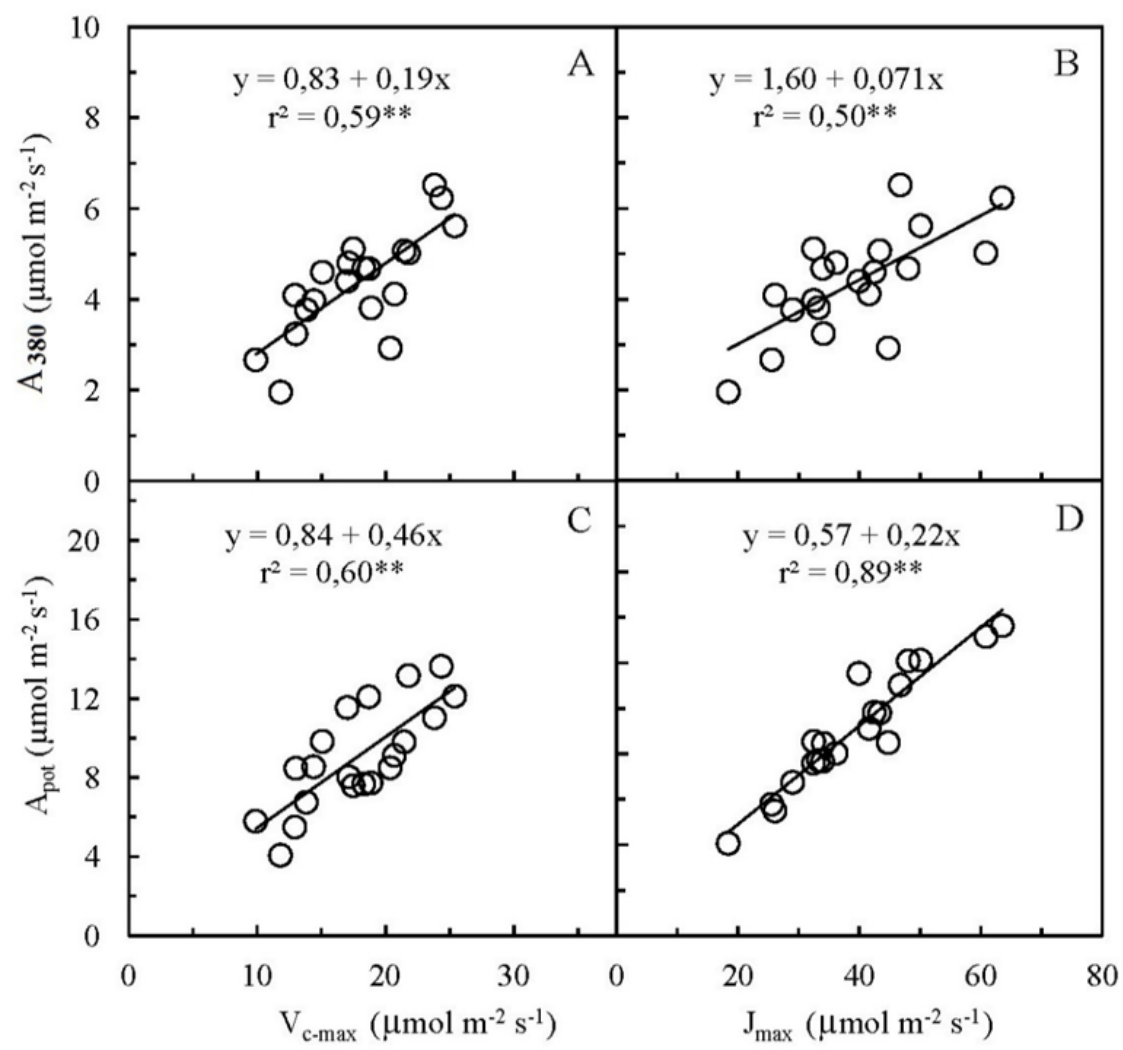

FIGURA 2: Taxas de fotossíntese $\mathrm{A}_{380}$ e $\mathrm{A}_{\text {pot }}$ em função da velocidade máxima de carboxilação da Rubisco $\left(\mathrm{V}_{\mathrm{c}-\max }\right)$ e taxa máxima de transporte de elétrons $\left(\mathrm{J}_{\max }\right)$ em cinco espécies da Amazônia central. Cada ponto representa uma planta por espécie. **: significativo a $1 \%$ de probabilidade. Os dados foram coletados de setembro a novembro de 2010. Valores de $\mathrm{J}_{\max }$ e $\mathrm{V}_{\mathrm{c} \text {-max }}$ foram padronizados para $25^{\circ} \mathrm{C}$. Medições foram feitas em $\left[\mathrm{CO}_{2}\right]$ de $\mathrm{CO}_{2}$ de $380 \mu \mathrm{mol} \mathrm{mol}^{-1}\left(\mathrm{~A}_{380}\right)$ e $2000 \mu \mathrm{mol} \mathrm{mol}{ }^{-1}\left(\mathrm{~A}_{\mathrm{pot}}\right)$, temperatura foliar de $27 \pm 2^{\circ} \mathrm{C}$ e irradiância de $1000 \mu \mathrm{mol} \mathrm{m} \mathrm{m}^{-2} \mathrm{~s}^{-1}$.

FIGURE 2: Photosynthetic rates $\mathrm{A}_{380}$ and $\mathrm{A}_{\text {pot }}$ as a function of the maximum carboxylation velocity of Rubisco $\left(\mathrm{V}_{\mathrm{c} \text {-max }}\right)$ and maximum electron transport rate $\left(\mathrm{J}_{\max }\right)$ in saplings of five species of the central Amazon. Each point represents one plant per species. **: significant at $1 \%$ probability. Data collected from September to November, 2010. Values of $\mathrm{J}_{\max }$ and $\mathrm{V}_{\mathrm{c} \text {-max }}$ were standardized to $25^{\circ} \mathrm{C}$. Measurements were made at a $\left[\mathrm{CO}_{2}\right]$ of $380\left(\mathrm{~A}_{380}\right)$ and $2000 \mu \mathrm{mol} \mathrm{mol}^{-1}\left(\mathrm{~A}_{\mathrm{pot}}\right)$, leaf temperature of $27 \pm 2{ }^{\circ} \mathrm{C}$ and irradiance of $1000 \mathrm{~mol} \mathrm{~m}^{-2} \mathrm{~s}^{-1}$.

Os valores de $\Gamma$ (média de $\left.79,3 \mu \mathrm{mol} \mathrm{mol}^{-1}\right)$ e $k_{\mathrm{x}}\left(22,2 \mathrm{mmol} \mathrm{m} \mathrm{m}^{-2} \mathrm{~s}^{-1}\right.$ ) relatados nesse estudo são menores do que aqueles encontrados por Marenco, Gonçalves e Vieira (2001) em árvores de diferentes fases sucessionais na Amazônia Central. A diferença pode ser atribuída ao fato de Marenco, Gonçalves e Vieira (2001) terem utilizados plantas aclimatadas a maiores níveis de irradiância. Os valores de $\mathrm{V}_{\mathrm{c} \text {-max }} \mathrm{e} \mathrm{J}_{\max }$ correlacionaram significativamente $(p<0,01)$ com as taxas de fotossíntese $\left(\mathrm{A}_{380}\right.$ e $\mathrm{A}_{\mathrm{pot}} ;$ Figura 2). O maior coeficiente de determinação foi observado para $\mathrm{A}_{\text {pot }}-\mathrm{J}_{\max }$ (Figura 2D), indicando que em altas concentrações de $\mathrm{CO}_{2}$ o transporte de elétrons é mais limitante que $\mathrm{V}_{\mathrm{c} \text {-max }}$. A relação entre $\mathrm{A}_{380}, \mathrm{~A}_{\mathrm{pot}}, \mathrm{V}_{\mathrm{c}-\max }$ e $\mathrm{J}_{\max }$ tem sido documentada em estudos anteriores (EVANS, 1983; EVANS; TERASHIMA, 1988). O maior coeficiente de determinação para a relação $\mathrm{A}_{380}-\mathrm{V}_{\mathrm{c}-\max }\left(\mathrm{r}^{2}=0,59 * *\right.$; Figura $\left.2 \mathrm{~A}\right)$ do que para $\mathrm{A}_{380}-\mathrm{J}_{\max }\left(\mathrm{r}^{2}=50^{* *}\right.$, Figura 2B) 
ocorre porque $\mathrm{V}_{\mathrm{c} \text {-max }}$ depende essencialmente dos valores das taxas de fotossíntese em baixas concentrações de $\mathrm{CO}_{2}$; já o $\mathrm{J}_{\max }$ tem como base as taxas de fotossíntese em concentração elevadas de $\mathrm{CO}_{2}$, muito acimas daquelas às quais a folha está normalmente exposta.

Na Figura 3, o ponto de intersecção das linhas contínua (que representa a taxa de carboxilação, $A_{c}$ ) e tracejada (que representa a taxa de transporte de elétrons, $A_{j}$ ) representa o ponto de colimitação (indicado pela linha vertical na Figura 3) ou ponto de equilíbrio, no qual a fotossíntese é igualmente limitada tanto pela taxa de carboxilação como pelo transporte de elétrons. $\mathrm{O}$ aumento de $\mathrm{C}_{\mathrm{i}}$ causou $\mathrm{o}$ aumento da fotossíntese nas espécies estudadas, até o ponto da limitação da fotossíntese pela taxa transporte de elétrons (linha tracejada na Figura 3). Abaixo do ponto de colimitação (valores de $C_{i}$ de 300 a 569 $\mu \mathrm{mol} \mathrm{mol}{ }^{-1}$, indicados pela linha vertical na Figura 3), tanto a taxa de carboxilação como o transporte de elétrons parecem limitar as taxas de fotossíntese. Entretanto, uma análise mais detalhada permite

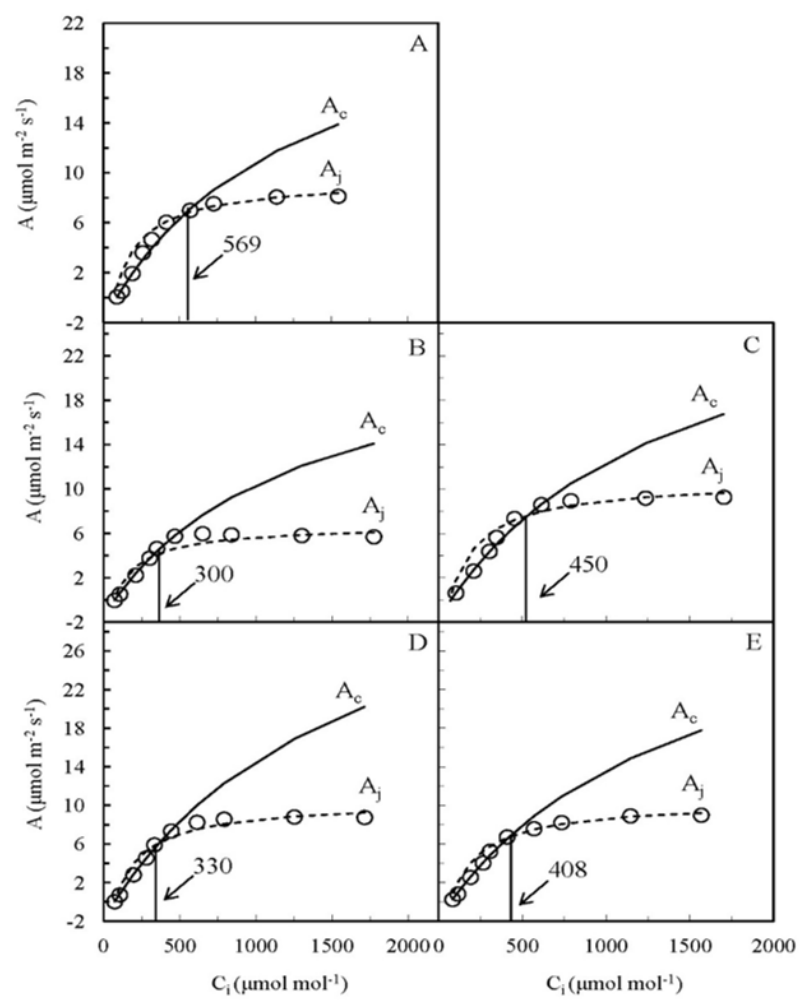

FIGURA 3: Taxa fotossintética em função da $\left[\mathrm{CO}_{2}\right]$ intercelular $\left(\mathrm{C}_{\mathrm{i}}\right)$ para Amphirrhox longifolia (A), Duguetia flagellaris (B), Fusaea longifolia (C), Guarea carinata (D) e Myrcia subsericea (E). A linha sólida mostra a limitação da fotossíntese imposta pela taxa de carboxilação da Rubisco ( $\mathrm{A}_{c}$ ). A linha tracejada indica a limitação da fotossíntese imposta pelo transporte de elétrons $\left(\mathrm{A}_{\mathrm{j}}\right)$. A linha contínua vertical mostra o valor de $\mathrm{C}_{\mathrm{i}}$ em que ocorre a transição da fotossíntese limitada por $\mathrm{A}_{\mathrm{c}}$ para fotossíntese limitada por $\mathrm{A}_{\mathrm{j}}$. Os números (nos gráficos) mostram os valores de $\mathrm{C}_{\mathrm{i}}$ em $\mu \mathrm{mol} \mathrm{mol}{ }^{-1}$. Cada ponto corresponde à média de quatro plantas por espécie. Os dados foram coletados de setembro a novembro de 2010.

FIGURE 3: Photosynthetic rate as a function of the intercellular $\left[\mathrm{CO}_{2}\right]$ concentration $\left(\mathrm{C}_{\mathrm{i}}\right)$ in Amphirrhox longifolia (A), Duguetia flagellaris (B), Fusaea longifolia (C), Guarea carinata (D) and Myrcia subsericea (E). The solid line shows the limitation of photosynthetic imposed by the carboxylation rate of Rubisco $\left(A_{c}\right)$. The dashed line indicates the limitation the photosynthetic imposed by electron transport $\left(\mathrm{A}_{\mathrm{i}}\right)$. The vertical solid line shows the value of $\mathrm{C}_{\mathrm{i}}$ at which occurs the transition from photosynthesis limited by $\mathrm{A}_{\mathrm{c}}$ to photosynthesis limited by $A_{j}$. The numerals (in the graphs) show the values for $C_{i}$ in $\mu$ mol mol ${ }^{-1}$. Each point represents the mean of four plants per species. Data were collected from September to November of 2010 . 
visualizar que à esquerda da linha vertical da Figura 3, as linhas que representam a taxa de carboxilação (linha contínua $-\mathrm{A}_{\mathrm{c}}$; Figura 3) coincidem exatamente com os valores observados. Isso indica que, abaixo do ponto de colimitação, as taxas fotossintéticas foram limitadas por $\mathrm{V}_{c-\max }$ indicando menor investimento em proteínas associadas à Rubisco (VALLADARES et al., 2002; ELLSWORTH et al., 2004; ONODA; HIKOSAKA; HIROSE, 2005). Os valores de $\mathrm{CO}_{2}$ no sub-bosque da floresta normalmente oscilam entre 420-450 $\mu \mathrm{mol} \mathrm{mol}^{-1}$ (MARENCO; NASCIMENTO; MAGALHÃES, 2014). Isto significa que nas condições ambientais normalmente encontradas no sub-bosque, a fotossíntese é limitada pela atividade da Rubisco. Nas curvas $\mathrm{A} / \mathrm{C}_{\mathrm{i}}$ observou-se uma variação interespecífica nos pontos de colimitação da fotossíntese. Estas diferenças influenciaram nos valores de $\mathrm{V}_{\mathrm{c} \text {-max }}$. Isto mostra a importância de se efetuar mais estudos para melhor caracterização das variações interespecíficas de espécies florestais da Amazônia.

Não houve diferença significativa nos valores de $\mathrm{V}_{c-\max }$ e $\mathrm{J}_{\max }$ entre os horários do dia (Figura 4) nem efeito significativo na interação entre espécie e horário do dia $(p>0,05)$, por isso, uma média das espécies é apresentada. Alguns estudos têm mostrado que a atividade da Rubisco oscila durante o dia apresentando baixa atividade nos horários próximos ao meio-dia (PARRY et al., 1993; HRSTKA et al., 2007), o que não ocorreu neste estudo. Isto mostra que fatores que normalmente influenciam o funcionamento dos estômatos (MARENCO; NASCIMENTO; MAGALHÃES, 2014) não têm o mesmo efeito na carboxilação

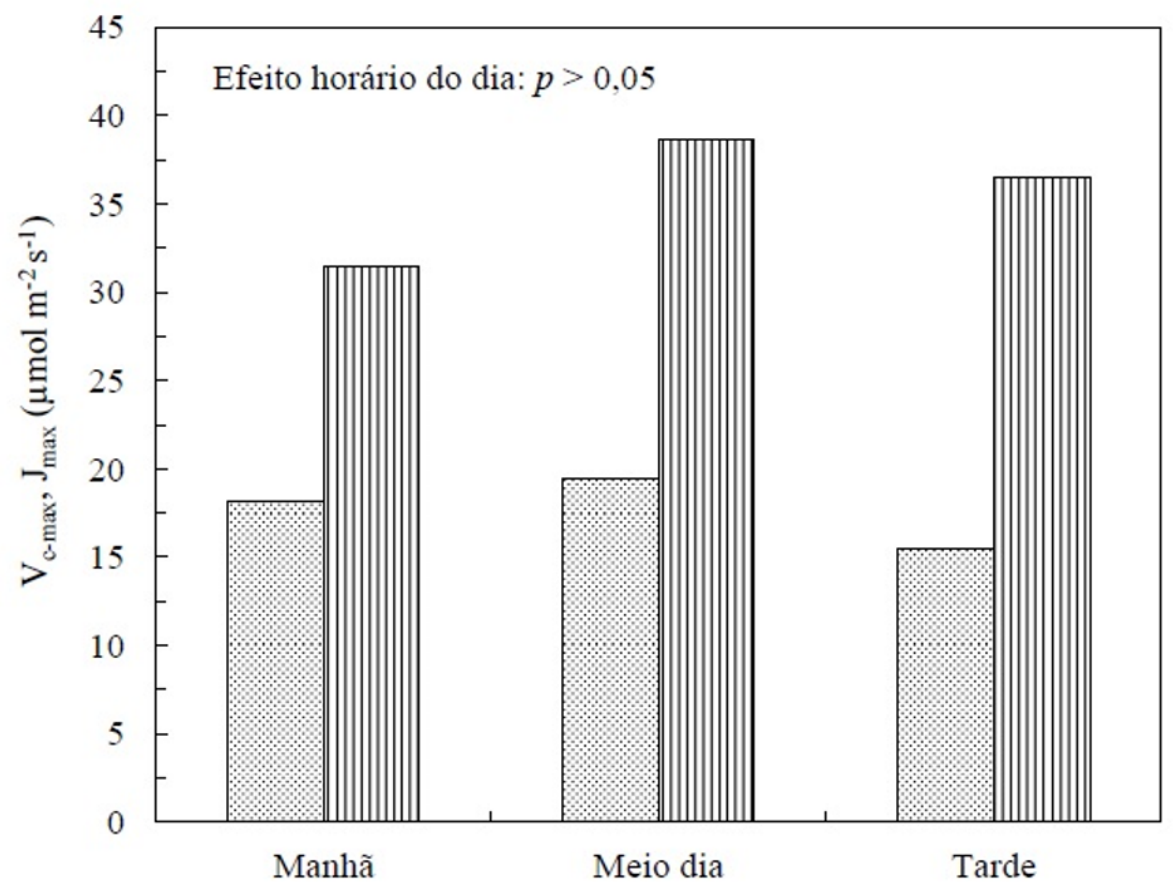

FIGURA 4: Velocidade máxima de carboxilação da Rubisco ( $\mathrm{V}_{\mathrm{c}-\max }$; barras pontilhadas) e taxa máxima de transporte de elétrons $\left(\mathrm{J}_{\max }\right.$; barras listradas) em função do horário do dia em cinco espécies da Amazônia central. Cada barra representa a média de cinco espécies e quatro plantas por espécie. Valores de $\mathrm{V}_{\mathrm{c} \text {-max }}$ e $\mathrm{J}_{\max }$ foram padronizados para $25^{\circ} \mathrm{C}$. Os dados foram coletados de setembro a novembro de 2010. Os valores de $\mathrm{V}_{\mathrm{c} \text {-max }}$ e $\mathrm{J}_{\max }$ durante o dia não diferem a $5 \%$ de probabilidade de acordo com o teste F.

FIGURE 4: Maximum carboxylation velocity of Rubisco $\left(\mathrm{V}_{\mathrm{c}-\max }\right.$; dotted bars) and maximum electron transport rate $\left(\mathrm{J}_{\max }\right.$; striped bar) as a function of time of day in saplings of five species of the central Amazon. Each bar represents the mean of five species and four plants per species. $\mathrm{V}_{\mathrm{c}-\max }$ and $\mathrm{J}_{\max }$ were standardized to $25^{\circ} \mathrm{C}$. Data were collected from September to November of 2010. Values of $\mathrm{V}_{\mathrm{c}-\max }$ and $\mathrm{J}_{\max }$ during daytime do not differ at $5 \%$ probability according the F-test. 
e no transporte de elétrons em arvoretas do sub-bosque e como resultado $\mathrm{V}_{\mathrm{c} \text {-max }}$ e $\mathrm{J}_{\max }$ tendem a permanecer estáveis durante a maior parte do dia (08h00-16h00), o que constitui uma vantagem adaptativa importante. Dessa forma, a planta pode aproveitar com eficiência os feixes ocasionais de luz direta que atingem o subbosque da floresta.

$\mathrm{O}$ aumento da irradiância e da temperatura do ar no sub-bosque levaram a um acréscimo em $\mathrm{A}_{380}$, $\mathrm{A}_{\mathrm{pot}}, \mathrm{V}_{\mathrm{c} \text {-max }}$ e $\mathrm{J}_{\max }$ (Figuras 5 e 6). Isto sugere maior alocação de recursos da folha para os complexos proteicos diretamente relacionados com a fotossíntese em plantas que recebem mais luz. Estes resultados concordam com outros estudos que relatam que a atividade da Rubisco responde a vários fatores ambientais, tais como irradiância (PONS; WELSCHEN, 2003) e a temperatura (WARREN; DREYE, 2006; WARREN, 2008; LLOYD; FARQUHAR, 2008). O aumento da temperatura dentro de uma faixa moderada $\left(28-36^{\circ} \mathrm{C}\right)$ pode aumentar $\mathrm{V}_{\mathrm{c}-\max }$ e $\mathrm{J}_{\max }$, refletindo um efeito positivo nas taxas de fotossíntese $\left(\mathrm{A}_{380}\right.$ e $\left.\mathrm{A}_{\text {pot }}\right)$. Por outro lado, o aquecimento foliar exacerbado pode ocasionar diminuição da eficiência de carboxilação da Rubisco ao promover a fotorrespiração (MARENCO et al., 2014).
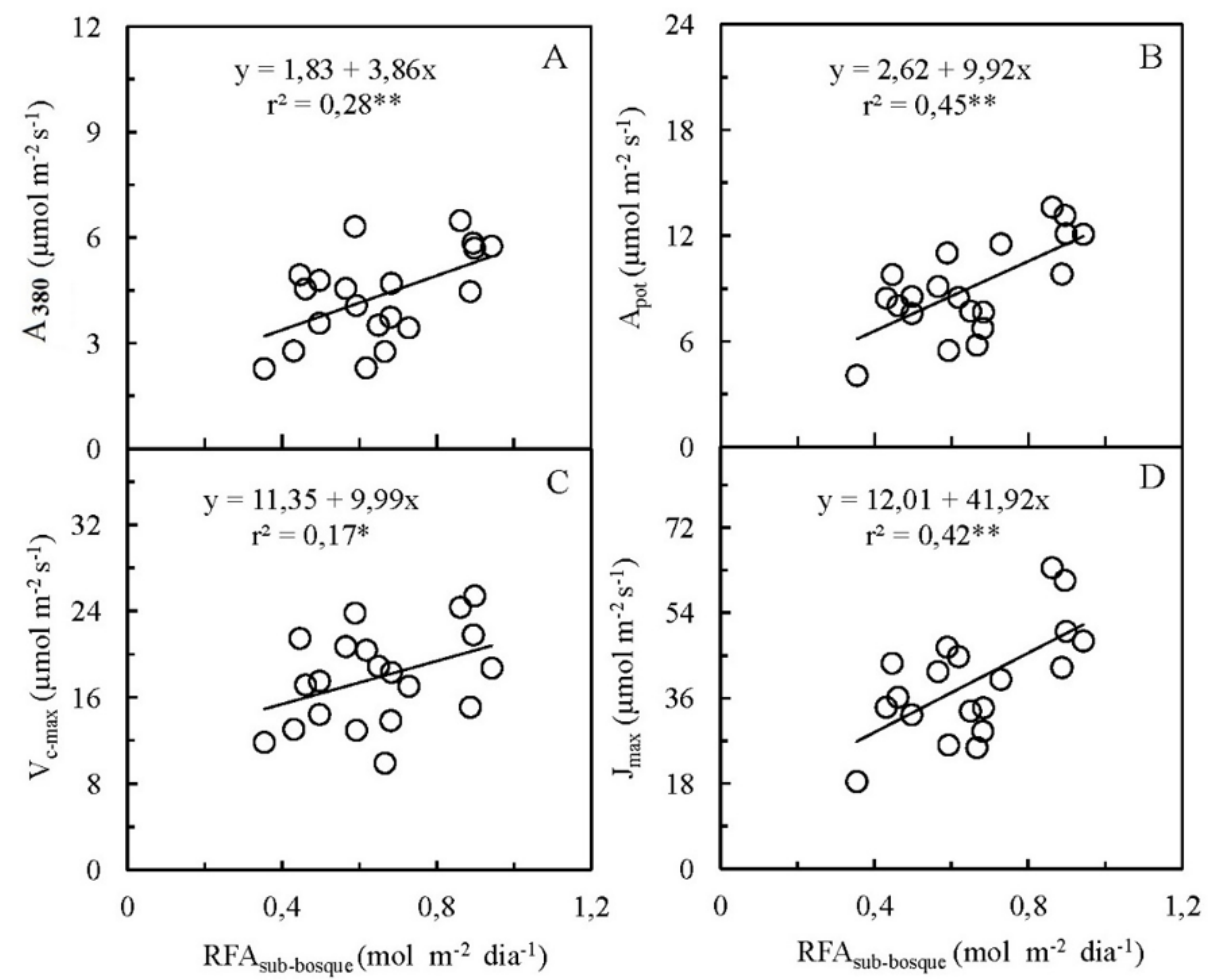

FIGURA 5: Fotossíntese em $380 \mu \mathrm{mol} \mathrm{mol}{ }^{-1}\left(\mathrm{~A}_{380}, \mathrm{~A}\right)$, capacidade fotossintética $\left(\mathrm{A}_{\text {pot }}\right.$, B), velocidade máxima de carboxilação da Rubisco $\left(\mathrm{V}_{\mathrm{c} \text {-max }}, \mathrm{C}\right)$ e taxa máxima de transporte de elétrons $\left(\mathrm{J}_{\max }\right.$, D) em função da radiação fotossinteticamente ativa no sub-bosque $\left(\right.$ RFA $\left._{\text {sub-bosque }}\right)$. Os dados de irradiância foram registrados durante as medições de trocas gasosas. Cada ponto representa uma planta por espécie. *: significativo a $5 \%$ de probabilidade. ${ }^{* *}$ : significativo a $1 \%$ de probabilidade. Valores de $\mathrm{V}_{\mathrm{c} \text {-max }}$ e $\mathrm{J}_{\max }$ foram padronizados para $25^{\circ} \mathrm{C}$. Os dados foram coletados de setembro a novembro de 2010. Condições do experimento conforme descrito na Figura 2.

FIGURE 5: Photosynthesis at $380 \mu \mathrm{mol} \mathrm{mol}{ }^{-1}\left(\mathrm{~A}_{380}, \mathrm{~A}\right)$, photosynthetic capacity $\left(\mathrm{A}_{\text {pot }}, \mathrm{B}\right)$, maximum carboxylation velocity of Rubisco $\left(\mathrm{V}_{\mathrm{c}-\max }, \mathrm{C}\right)$ and maximum electron transport rate $\left(\mathrm{J}_{\max }, \mathrm{D}\right)$ as a function of photosynthetically active radiation in the understory (RFA sub-bosque $)$. Irradiance data were recorded at the time of gas exchange measurements. Each point represents one plant per species. *: significant at $5 \%$ probability. ${ }^{* *}$ : significant at $1 \%$ probability. $\mathrm{V}_{\mathrm{c} \text {-max }}$ and $\mathrm{J}_{\max }$ were standardized to $25^{\circ} \mathrm{C}$. Data collected from September to November of 2010. Conditions of the experiment are as described in Figure 2. 

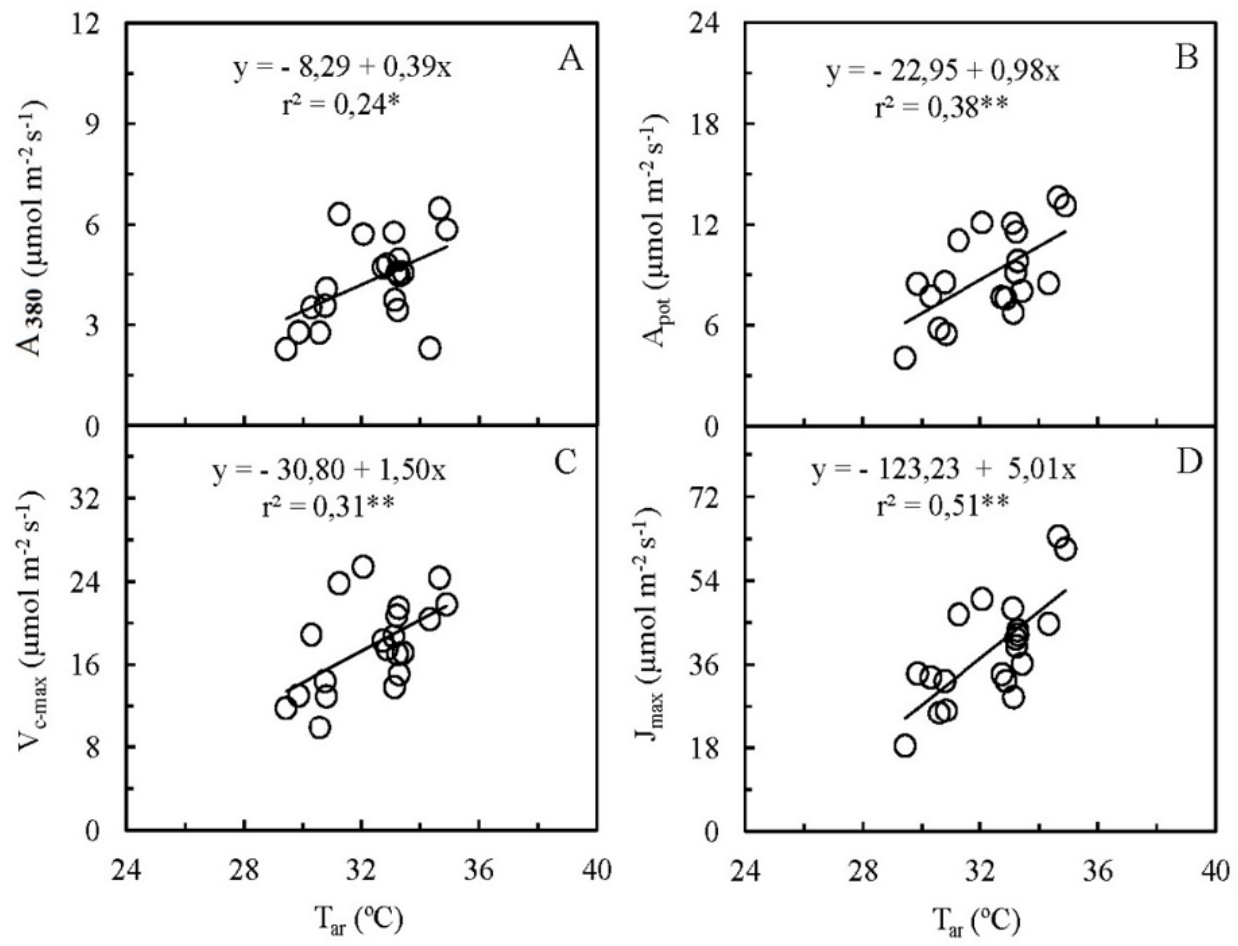

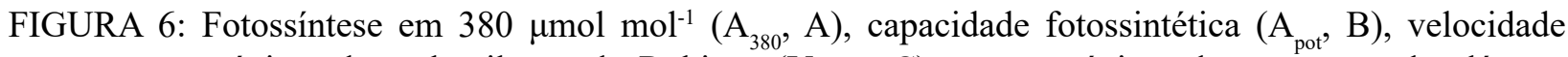
máxima de carboxilação da Rubisco $\left(\mathrm{V}_{\mathrm{c}-\max }, \mathrm{C}\right)$ e taxa máxima de transporte de elétrons $\left(\mathrm{J}_{\max }, \mathrm{D}\right)$ em função da temperatura do $\operatorname{ar}\left(\mathrm{T}_{\mathrm{ar}}\right)$. Os dados de temperatura foram registrados durante as medições das trocas gasosas. Cada ponto representa uma planta por espécie. $*$ : significativo a $5 \%$ de probabilidade. ${ }^{* *}$ : significativo a $1 \%$ de probabilidade. Valores de $\mathrm{J}_{\max } \mathrm{eV}_{\mathrm{c} \text {-max }}$ foram padronizados para $25^{\circ} \mathrm{C}$. Os dados foram coletados de setembro a novembro de 2010. Condições do experimento conforme descrito na Figura 2.

FIGURE 6: Photosynthesis at $380 \mu \mathrm{mol} \mathrm{mol}{ }^{-1}\left(\mathrm{~A}_{380}, \mathrm{~A}\right)$, photosynthetic capacity $\left(\mathrm{A}_{\mathrm{pot}}, \mathrm{B}\right)$, maximum carboxylation velocity of Rubisco $\left(\mathrm{V}_{\mathrm{c}-\max }, \mathrm{C}\right)$ and maximum electron transport rate $\left(\mathrm{J}_{\max }, \mathrm{D}\right)$ as a function of air temperature $\left(\mathrm{T}_{\mathrm{ar}}\right)$. Temperature data were recorded during gas exchange measurements. Each point represents one plant per species. *: significant at 5\% probability. **: significant at $1 \%$ probability. $\mathrm{V}_{\mathrm{c} \text {-max }}$ and $\mathrm{J}_{\max }$ were standardized to $25^{\circ} \mathrm{C}$. Data were collected from September to November of 2010. Conditions of the experiment are as described in Figure 2.

\section{CONCLUSÕES}

Os parâmetros fotossintéticos $\left(\mathrm{V}_{\mathrm{c} \text {-max }}\right.$ e $\left.\mathrm{J}_{\max }\right)$ foram responsivos às variações na irradiância e temperatura no sub-bosque. Porém, $\mathrm{V}_{\mathrm{c}-\max }$ e $\mathrm{J}_{\max }$ não responderam de forma direta ao horário do dia, possibilitando, dessa forma, melhor aproveitamento dos feixes ocasionais de luz direta que atingem o sub-bosque da floresta ao longo do dia.

Em alta concentração de $\mathrm{CO}_{2}$, a assimilação de carbono é limitada especificamente por $J_{\max }$, o que reflete na fotossíntese potencial; portanto, o efeito do aumento da concentração atmosférica de $\mathrm{CO}_{2}$ na fotossíntese pode ser restringido por $J_{\max }$. Avaliações complementares da fisiologia dessas espécies são ainda necessárias, particularmente sob atmosfera enriquecida com $\mathrm{CO}_{2}$, uma vez que podem ocorrer respostas de aclimatação em longo prazo. 


\section{AGRADECIMENTOS}

Ao MCTI-INPA, à Fundação de Amparo à Pesquisa do Estado do Amazonas - FAPEAM (projeto UA 062.03164/2012) e Conselho Nacional de Desenvolvimento Científico e Tecnológico (CNPq) e CAPES pela concessão de bolsas aos participantes envolvidos na pesquisa.

\section{REFERÊNCIAS}

ALARCÓN, J. G. S. Levantamento florístico e etnobotânico em um hectare de floresta de terra firme na região do Médio Rio Negro, Roraima, Brasil. 2005. 111 f. Dissertação (Mestrado) - Instituto de Pesquisas Jardim Botânico do Rio de Janeiro, Rio de Janeiro, 2005.

ANDRADE, S. O. et al. Inflammatory action and toxicity of Guarea trichilioides L. in rats. Toxicology and Applied Pharmacology, New Mexico, v. 38, p. 39-46, 1976.

BOARDMAN, N. K. Comparative photosynthesis of sun and shade plants. Annual Review of Plant Physiology, Palo Alto, v. 28, p. 355-377, 1977.

CAMACHO, M. D. R. et al. Terpenoids from Guarea rhophalocarpa. Phytochemistry, London, v. 56, p. 203-210, 2001.

CHATROU, L. W.; HE, P. Studies in Annonaceae XXXIII. A revision of Fusaea (Baill.) Saff. Brittonia, New York, v. 52, p. 181-203, 1999.

ELLSWORTH, D. et al. Photosynthesis, carboxylation and leaf nitrogen responses of 16 species to elevated $\mathrm{CO}_{2}$ across four free-air $\mathrm{CO}_{2}$ enrichment experiments in forest, grassland and desert. Global Change Biology, Champaign, v. 10, p. 2121-2138, 2004.

EVANS, J. R. Nitrogen and photosynthesis in the flag leaf of wheat (Triticum aestivum L.). Plant Physiology, United Kingdom, v. 72, p. 297-302, 1983.

EVANS, J. R.; TERASHIMA, I. Photosynthetic characteristics of spinach leaves grown with different nitrogen treatment. Plant Cell Physiology, United Kingdom, v. 29, p. 157-165, 1988.

FARQUHAR, G. D.; VON CAEMMERER, S.; BERRY, J. A. A biochemical model of photosynthetic $\mathrm{CO}_{2}$ assimilation in leaves of $\mathrm{C}_{3}$ species. Planta, Berlin, v. 149, p. 78-90, 1980.

FECHINE, I. M. et al. Alkaloids and volatile constituents from Duguetia flagellaris. Biochemical Systematics and Ecology, Oxford, v. 30, p. 267-269, 2002.

FETCHER, N.; OBERBAUER, S. F.; CHAZDON, R. L. Physiological ecology of plants. In: MCDADE, L. A. et al. (Eds.). La Selva: ecology and natural history of a neotropical rain forest. Chicago: The University of Chicago Press, 1994. p. 128-141.

HRSTKA, M. et al. Diurnal regulation of ribulose-1,5-bisphosphate carboxylase/oxygenase activity and its content in Norway spruce needles. Photosynthetica, Praha, v. 45, p. 334-339, 2007.

JADÁN, O. et al. Riqueza y potencial maderable em sistemas agroforestales tradicionales como alternativa al uso del bosque nativo, Amazonia del Ecuador. Revista Forestal Mesoamericana Kurú, Cartago, v. 12, p. 13-22, 2015.

LIMA FILHO, D. A. et al. Aspectos florísticos de 13 hectares da área de Cachoeira Porteira-PA. Acta Amazonica, Manaus, v. 34, p. 415-423, 2004.

LLOYD, J.; FARQUHAR, G. D. Effects of rising temperatures and $\left[\mathrm{CO}_{2}\right]$ on the physiology of tropical forest trees. Philosophical Transactions of the Royal Society of Biological Sciences, London, v. 363, p. 1811-1817, 2008.

LOPEZ-TOLEDO, L. et al. Soil and light effects on the sapling performance of the shade-tolerant species Brosimum alicastrum (Moraceae) in a Mexican tropical rain forest. Journal of Tropical Ecology, Cambridge, v. 24, p. 629-637, 2008.

MALHI, Y.; WRIGHT, J. Spatial patterns and recent trends in the climate of tropical rainforest regions. Philosophical Transactions of the Royal Society of London, London, v. 359, p. 311-329, 2004.

MANTER, D. K.; KERRIGAN, J. $A / C_{i}$ curve analysis across a range of woody plant species: influence of regression analysis parameters and mesophyll conductance. Journal of Experimental Botany, United 
Kingdom, v. 55, p. 2581-2588, 2004.

MARENCO, R. A. et al. Fisiologia de espécies florestais da Amazônia: fotossíntese, respiração e relações hídricas. Revista Ceres, Viçosa, MG, v. 61, p. 786-799, 2014.

MARENCO, R. A. et al. Juvenile tree growth correlates with photosynthesis and leaf phosphorus content in central Amazonia. Revista Ceres, Viçosa, MG, v. 62, p. 175-183, 2015.

MARENCO, R. A.; GONÇALVES, J. F. C.; VIEIRA, G. Leaf gas exchange and carbohydrates in tropical trees differing in successional status in two light environments in central Amazonia. Tree Physiology, Oxford, UK, v. 21, p. 1311-1318, 2001.

MARENCO, R. A.; NASCIMENTO, H. C. S.; MAGALHÃES, N. S. Stomatal conductance in Amazonian tree saplings in response to variations in the physical environment. Photosynthetica, Praha, v. 52, p. 493500, 2014.

MARTINS, R. C. C. et al. Constituents of the essential oil of Eugenia nitida Camb. (Myrtaceae). Journal of Essential Oil Research, Carol Stream, v. 11, p. 724-726, 1999.

MEDLYN, B. E. et al. Effects of elevated $\left[\mathrm{CO}_{2}\right]$ on photosynthesis in European forest species: a meta-analysis of model parameters. Plant Cell and Environment, New York, v. 22, p. 1475-1495, 1999.

MENDES, K. R.; MARENCO, R. A. Leaf traits and gas exchange in saplings of native tree species in the Central Amazon. Scientia Agricola, Piracicaba, v. 67, p. 624-632, 2010.

MENDES, K. R.; MARENCO, R. A. Is stomatal conductance of Central Amazonian saplingsinfluenced by circadian rhythms under natural conditions? Theoretical and Experimental Plant Physiology, Campos dos Goytacazes, v. 26, p. 115-125, 2014.

MENDES, K. R.; MARENCO, R. A. Photosynthetic traits of tree species in response to leaf nutrient content in the central Amazon. Theoretical and Experimental Plant Physiology, Campos dos Goytacazes, v. 27, p. 51-59, 2015.

MILLER, R. P.; WANDELLI, E. V.; GRENAND, P. Conhecimento e utilização da floresta pelos índios waimiri-atroari do rio camanau - Amazonas. Acta Botanica Brasílica, Porto Alegre, v. 3, p. 47-56, 1989.

NASCIMENTO, H. C. S.; MARENCO, R. A. Mesophyll conductance variations in response to diurnal environmental factors in Myrcia paivae and Minquartia guianensis in Central Amazonia. Photosynthetica, Praha, v. 51, p. 457-464, 2013.

ONODA, Y.; HIKOSAKA, K.; HIROSE, T. Seasonal change in the balance between capacities of RuBP carboxylation and RuBP regeneration affects $\mathrm{CO}_{2}$ response of photosynthesis in Polygonum cuspidatum. Journal of Experimental Botany, United Kingdom, v. 56, p. 755-763, 2005.

PARRY, M. A. J. et al. Water stress and the diurnal activity of ribulose-1, 5-bisphosphate carboxylase in field grown Nicotiana tabacum genotypes selected for survival at low $\mathrm{CO}_{2}$ concentrations. Plant Physiology and Biochemistry, Paris, v. 31, p. 113-120, 1993.

PONS, T. L.; WELSCHEN, R. A. M. Midday depression of net photosynthesis in the tropical rainforest tree Eperua grandiflora: contributions of stomatal and internal conductances, respiration and Rubisco functioning. Tree Physiology, Oxford, UK, v. 23, p. 937-947, 2003.

RESQUE, O. R. Vocabulário de frutas comestíveis na Amazônia. Belém: Museu Paraense Emílio Goeld, 2007. $99 \mathrm{p}$.

RODRIGUES, P. A. Avaliação de extratos vegetais bioativos de plantas amazônicas sobre a concentração de testosterona sérica em camundongos pós púberes. 2013. 77 f. Dissertação (Mestrado) - Universidade Paulista, São Paulo, 2013.

SELLERS, P. J. et al. Modeling the exchanges of energy, water, and carbon between continents and the atmosphere. Science, Washington, v. 275, p. 502-509, 1997.

TAVARES, J. F. et al. Alkaloids and volatile constituents from the stem of Fusaea longifolia (Aubl.) Saff. (Annonaceae). Brazilian Journal of Pharmacognosy, Curitiba, v. 15, p. 115-118, 2005.

VALLADARES, F. et al. The greater seedling high-light tolerance of Quercus robur over Fagus sylvatica is linked to a greater physiological plasticity. Trees Structure and Function, Berlin, v. 16, p. 395-403, 2002. VALLADARES, F.; NIINEMETS, Ü. Shade tolerance, a key plant feature of complex nature and consequences. Annual Review of Ecology Evolution and Systematics, Palo Alto, v. 39, p. 237-257, 2008. WARREN, C. R. Soil water deficits decrease the internal conductance to $\mathrm{CO}_{2}$ transfer but atmospheric water deficits do not. Journal of Experimental Botany, United Kingdom, v. 59, p. 327-334, 2008. 
WARREN, C. R.; DREYE, E. Temperature response of photosynthesis and internal conductance to $\mathrm{CO}_{2}$ : results from two independent approaches. Journal of Experimental Botany, United Kingdom, v. 57, p. 3057-3067, 2006.

WAY, D. A.; OREN, R. Differential responses to changes in growth temperature between trees from different functional groups and biomes: a review and synthesis of data. Tree Physiology, Oxford, UK, v. 30, p. 669-688, 2010.

WULLSCHLEGER, S. D. Biochemical limitations to carbon assimilation in $\mathrm{C}_{3}$ plants - a retrospective analysis of the $A / C_{\mathrm{i}}$ curves from 109 species. Journal of Experimental Botany, United Kingdom, v. 44, p. $907-920,1993$. 